\title{
CONTINUOUS LINEAR OPERATORS ON SPACES OF CONTINUOUS FUNCTIONS
}

\author{
H. ELTON LACEY AND PETER D. MORRIS ${ }^{1}$
}

Throughout this paper $X$ denotes a bicompact space, $B(X)$ denotes the Banach space of all bounded real-valued functions on $X, C(X)$ denotes the closed linear subspace of $B(X)$ of continuous real-valued functions on $X, B(N)$ is denoted by $m$ (where $N$ is the set of positive integers). The space $l_{1}(X)$ is the Banach space of all absolutely summable real-valued functions on $X$ and $l_{1}$ denotes $l_{1}(N)$. The sequence Banach spaces $c_{0}$ and $l_{p}(1<p<\infty)$ are respectively the spaces of all real sequences converging to 0 and all $p$ th absolutely summable sequences. The space $L_{1}$ is the Banach space of real-valued Lebesgue integrable functions on $[0,1]$. If $E$ is a Banach space, then $E^{*}, E^{* *}$ denote the first and second duals of $E$ respectively. The weak topology on $E$ is signified by $\sigma\left(E, E^{*}\right)$ and the weak* topology on $E^{*}$ is signified by $\sigma\left(E^{*}, E\right)$. If $E$ and $F$ are Banach spaces and $T$ is a continuous linear operator (hereafter called an operator) from $E$ to $F$, then $T^{*}$ denotes the dual operator from $F^{*}$ to $E^{*}$. If $T$ is on to $F$, then $F$ is said to be a continuous linear image (c.li.) of $E$. If $T$ maps a neighborhood of 0 in $E$ into a norm (weak) conditionally compact set in $F$, then $T$ is said to be compact (weakly compact). If $T$ maps weak Cauchy sequences in $E$ into norm convergent sequences in $F$, then $T$ is said to be completely continuous. If the restriction of $T$ to each infinite dimensional linear subspace of $E$ is not a topological linear isomorphism (hereafter called an isomorphism), then $T$ is said to be strictly singular.

The purpose of this paper is to study the classes of operators defined above when their domain is a c.l.i. of $C(X)$. In particular, necessary and sufficient conditions are given to make all four of the classes coincide and for them to coincide in pairs. It is shown in (3), (4), $(5),(6),(7)$ and (8) of Theorem 4 that any pair of the classes of operators coincide for domains all c.l.i. of $C(X)$ if and only if $X$ is dispersed. It is well known that if the domain of the operators is $C(X)$, then the completely continuous and the weakly compact operators coincide [4]. This result does not hold, in general, for a c.l.i. of $C(X)$ as is shown in this paper. Recently [15] it has been shown that if the

Received by the editors March 17, 1966.

1 This author was partially supported by NASA-MSC (Houston) contract NAS 9-3848. 
domain is a space $C(X)$, then the strictly singular and the weakly compact operators coincide. Again, it is shown in this paper that this result does not hold for a general c.l.i. of $C(X)$.

Lemma 1. If the Banach space $B$ is an abstract $(L)$-space and $E$ is a separable linear subspace of $B$, then (a) the smallest closed sublattice of $B$ containing $E$ is separable and hence $E$ is isomorphic to a subspace of $L_{1}$ [7, Theorems 8,11].

(b) if $E^{*}$ is separable, then $E$ is reflexive [5].

Lemma 2. If $E$ is a reflexive Banach space and $E^{*}$ is isomorphic to a subspace of the Banach space $F^{*}$, then $E$ is a c.l.i. of $F$.

Proposition 3. If $X$ has a perfect set and $E^{* *}$ is separable, then the following are equivalent.

(1) $E$ is reflexive and a c.l.i. of $C(X)$.

(2) $E^{*}$ is isomorphic to a subspace of $L_{1}$.

(3) $E$ is a c.l.i. of $m$.

Proof. If $E$ is a c.l.i. of $C(X)$, then $E^{*}$ is isomorphic to a separable subspace of $C(X)^{*}$. Since $C(X)^{*}$ is an abstract $(L)$-space, (2) follows from Lemma 1.

Conversely, if $E^{*}$ is isomorphic to a subspace of $L_{1}$, then since $E^{* *}$ is separable, $E^{*}$ and hence $E$ is reflexive. By Lemma 2 , to show that $E$ is a c.l.i. of $C(X)$ it suffices to show that $E^{*}$ is isomorphic to a subspace of $C(X)^{*}$. To do this it is shown that $L_{1}$ is isomorphic to a subspace of $C(X)^{*}$. (This is proven in [14]. An independent proof is given here and utilized later in the paper.) Since $X$ has a perfect set, an adaption of the proof of Urysohn's Lemma shows that there is a continuous on to map $h$ of $X$ on to [0,1] (see [14] also). Let $M$ be the linear subspace of $C(X)$ of all functions of the form $f \circ h$, where $f$ is in $C[0,1]$. Consider the positive linear functional $\mu$ defined on $M$ by $\mu(f \circ h)=\int_{0}^{1} f(t) d t$. By [8, p. 20] $\mu$ has a positive extension (called $\mu$ ) to all of $C(X)$. Let $N$ be the normed linear space whose elements are the elements of $C[0,1]$ but the norm is $\int_{0}^{1}|f(t)| d t$. Consider the operator $A$ from $N$ to $C(X)^{*}$ defined by $(A f)(g)=\int_{X} g(f \circ h) d \mu$ for each $g$ in $C(X)$ and $f$ in $C[0,1]$. Since $\mu$ is a positive Radon measure on $X$, $\|A f\|=\int_{X}|f \circ h| d \mu=\int_{0}^{1}|f(t)| d t=\|f\|$ and $A$ is an isometry. Hence $A$ has an extension from $L_{1}$ into $C(X)^{*}$ which is an isometry.

Since $m^{*}$ is an abstract $(L)$-space, the same argument as above shows that (3) implies (2).

Conversely, if $E^{*}$ is isomorphic to a subspace of $L_{1}$, then $E^{* *}$ is a c.l.i. of $\left(L_{1}\right)^{*}$. By Lemma $1, E=E^{* *}$ and by [12] $\left(L_{1}\right)^{*}$ is isomorphic to $m$. 
REMARK. By [10] $l_{p}$ is not isomorphic to a subspace of $L_{1}$ for $2<p<\infty$ and by [6] $l_{p}$ is isomorphic to a subspace of $L_{1}$ for $1 \leqq p \leqq 2$. Thus if $X$ has a perfect set, then $l_{p}$ is a c.l.i. of $C(X)$ for $2 \leqq p \leqq \infty$ and $l_{p}$ is not a c.l.i. of $C(X)$ for $1<p<2$. (The fact that $l_{2}$ is a c.l.i. of $C(X)$ when $X$ has a perfect set was communicated by A. Pelczynski in a letter to R. J. Whitley.)

Proposition 4. If $E$ and $F$ are Banach spaces and $E$ is a c.l.i. of $C(X)$, then

(1) each completely continuous operator from $E$ to $F$ is weakly compact,

(2) each strictly singular operator from $E$ to $F$ is weakly compact,

(3) each completely continuous operator from $E$ to $F$ is strictly singular.

The proof of (1) is immediate, (2) follows from the fact [15] that strictly singular operators with domain $C(X)$ are weakly compact, and (3) follows from (1) (see [9]).

A bicompact space $X$ is said to be dispersed if it contains no perfect subsets. An exhaustive study of dispersed spaces is made in [14] and several results of that paper are used frequently here.

THEOREM 5. The following statements are equivalent.

(1) The bicompact space $X$ is dispersed.

(2) Every reflexive c.l.i. of $C(X)$ is finite dimensional. For each c.l.i. E of $C(X)$ and each Banach space $F$,

(3) each weakly compact operator from $E$ to $F$ is compact,

(4) each completely continuous operator from $E$ to $F$ is compact,

(5) each strictly singular operator from $E$ to $F$ is compact,

(6) each weakly compact operator from $E$ to $F$ is completely continuous,

(7) each strictly singular operator from $E$ to $F$ is completely continuous,

(8) each weakly compact operator from $E$ to $F$ is strictly singular,

(9) each continuous linear operator from $E^{* *}$ to $F$ which is $\sigma\left(E^{* *}, E^{*}\right)$ to $\sigma\left(F, F^{*}\right)$ continuous is compact.

(10) For each c.l.i. E. of $C(X)$, each continuous linear operator from $E$ to $E^{*}$ is compact.

(11) Every Radon measure on $X$ has an extension to all the subsets of $X$.

Proof. The fact that if $X$ is not dispersed, then $l_{2}$ is a c.l.i. of $X$ (see Proposition 3 ) is used throughout. Using this fact it is immediate that (2) implies (1). Using Proposition 4 it is immediate that (5) 
implies (4), (6) implies (7), and (3) implies (5). Since an operator that is both weakly compact and completely continuous is strictly singular, (6) implies (8). If $E$ is reflexive, then the identity operator $E$ is weakly compact and thus (3) implies (2). Using the operator mapping $C(X)$ onto $l_{2}$ it is clear that (not 1) implies (not 4). If $E=l_{2}$ and $F=l_{p}(p>2)$ and $T$ is the injection of $l_{2}$ into $l_{p}$, then $T$ is strictly singular but not completely continuous and hence (not 1) implies (not 7). The identity map from $l_{2}$ to $l_{2}$ suffices to show that (not 1) implies (not 8). The implication (3) implies (6) is clear. After showing that (1) implies (3), it will have been established that (1) through (8) are equivalent. Let $A$ be a continuous linear map of $C(X)$ onto $E$ and $T$ be a weakly compact operator from $E$ to $F$. Then $T A$ is weakly compact and hence completely continuous. Let $\left(f_{n}\right)$ be a bounded sequence in $C(X)$. Then by [14] there is a weakly Cauchy subsequence $\left(f_{n_{j}}\right)$ of $\left(f_{n}\right)$. Therefore $\left(T A f_{n_{j}}\right)$ converges in norm and $T A$ is compact. It is immediate that $T$ is compact.

If $X$ is dispersed and $A$ is a continuous linear map of $C(X)$ onto $E$ and $T$ is a continuous linear operator from $E$ to $E^{*}$, then since $C(X)^{*}$ $=l_{1}(X)[14], A^{*} T$ is completely continuous and by (4) $A^{*} T$ is compact. Since $A^{*}$ is an isomorphism, $T$ is compact and (1) implies (10). Conversely, if $X$ is not dispersed, then $l_{2}$ is a c.l.i. of $X$ and $l_{2}$ is isomorphic to a subspace of $C(X)^{*}$. Hence (not 1) implies (not 10).

Suppose $X$ is dispersed and $E$ is a c.l.i. of $C(X)$. Let $T$ be a continuous linear operator from $E^{* *}$ to $F$ which is $\sigma\left(E^{* *}, E^{*}\right)$ to $\sigma\left(F, F^{*}\right)$ continuous. Then $T$ is weakly compact and thus $T$ restricted to $E$ is weakly compact. Thus by (3) $T$ restricted to $E$ is compact. Since the unit sphere of $E$ is $\sigma\left(E^{* *}, E^{*}\right)$ dense in the unit sphere of $E^{* *}$, it follows that $T$ is compact and thus (3) implies (9). On the other hand, if $X$ is not dispersed, then $l_{2}$ is a c.l.i. of $C(X)$ and the identity from $l_{2}$ to $l_{2}$ shows that (not 1) implies (not 9).

If $X$ is dispersed, then every Radon measure $\mu$ on $X$ is purely atomic [14]. That is, there is a sequence $\left(a_{n}\right)$ in $l_{1}$ and a sequence $\left(X_{n}\right)$ in $X$ such that if $B$ is a subset of $X$, then $\mu(B)=\sum_{X_{n} \in B} a_{n}$. Hence (1) implies (11).

Conversely, if $X$ is not dispersed, then as in the proof of (2) implies (1) of Proposition 3 there is a continuous map $h$ of $X$ onto $[0,1]$ and a positive Radon measure $\mu$ on $X$ such that $\int_{0}^{1} f(t) d t=\int_{X} f \circ h d \mu$ for all $f$ in $C[0,1]$. Thus the measure $\nu$ on the Borel sets of $[0,1]$ given by $\nu(B)=\mu\left[h^{-1}(B)\right]$ is Lebesque measure (since $\int_{X} f \circ h d \mu=\int_{[0,1]} f d \nu$ for all $f$ in $C[0,1])$. If $\mu$ had an extension to all the subsets of $X$, then $\nu$ would have an extension to all the subsets of $[0,1]$. Hence (not 1) implies (not 11). 
Proposition 5. (a) For each closed linear subspace $M$ of $c_{0}$ and each Banach space $F$, each strictly singular operator $T$ from $M$ to $F$ is weakly compact.

(b) For each c.l.i. E of $c_{0}$, each closed infinite dimensional subspace $M$ of $E$ contains a subspace isomorphic to $c_{0}$ (this generalizes Corollary 4 of $[11])$.

Proof. (a) By [4, p. 171] $T$ is weakly compact if and only if it is completely continuous. Thus it suffices to show that if $T$ is not completely continuous, then it is not strictly singular. Suppose $T$ is strictly singular and not completely continuous. Then there is a sequence $\left(f_{n}\right)$ in $M$ such that $\left(f_{n}\right)$ converges weakly to 0 , but $\left(T f_{n}\right)$ does not converge to 0 in norm. Thus it may be assumed that $\left\|f_{n}\right\|=1$ and that $\left(T f_{n}\right)$ is bounded away from 0 in norm. Now by $[1, \mathrm{p} .194]$ there is a subsequence $\left(f_{n_{j}}\right)$ of $\left(f_{n}\right)$ such that $c_{0}$ is isomorphic to the closed linear space $N$ of $\left(f_{n_{j}}\right)$. Moreover, $T$ restricted to $N$ is strictly singular and hence by (7) of Theorem $4, T$ restricted to $N$ is completely continuous. Hence $\left(T f_{n_{j}}\right)$ converges to 0 in norm, which is a contradiction.

(b) Let $E$ be a c.li. of $c_{0}$ and $A$ be a continuous linear operator from $c_{0}$ onto $E$. Suppose $M$ is a closed subspace of $E$ and $N=A^{-1}(M)$. Then the restriction $A_{0}$ of $A$ to $N$ is on to $M$. If $A_{0}$ is strictly singular, then by (a) $A_{0}$ is weakly compact and hence $M$ is finite dimensional [4, p. 171]. Thus if $M$ is infinite dimensional, then there is a closed infinite dimensional subspace $K$ of $N$ such that $A$ restricted to $K$ is an isomorphism. But, $K$ contains a subspace isomorphic to $c_{0}$.

\section{BIBLIOGRAPHY}

1. S. Banach, Théorie des opérations linéares, Chelsea, New York, 1955.

2. J. Dixmier, Sur un théorème de Banach, Duke Math. J. 15 (1948), 1057-1071.

3. N. Dunford and J. Schwartz, Linear operators. Part I, Interscience, New York, 1958.

4. A. Grothendieck, Sur les applications lineares faiblement compactes d'espaces du type $C(K)$, Canad. J. Math. 5 (1953), 129-173.

5. M. Kadec and A. Pelczynski, Bases, lacunary sequences and complemented subspaces in the spaces $L_{p}$, Studia Math. 21 (1962), 161-176.

6. M. J. Kadec, On linear dimension of the spaces $L_{p}$, Uspehi Mat. Nauk 11 (1954), 107-109. (Russian)

7. S. Kakutani, Concrete representations of abstract $(L)$-spaces and the meanergodic theorem, Ann. of Math. (2) 42 (1944), 523-537.

8. J. L. Kelley and I. Namioka, Linear topological spaces, Van Nostrand, New York, 1963.

9. H. E. Lacey and R. J. Whitley, Conditions under which all the bounded linear maps are compact, Math. Ann. 158 (1965), 1-5. 
10. R. E. A. C. Paley, On linear dimension of $l_{p}$ and $L_{p}$, Bull. Amer. Math. Soc. 42 (1936), 235-240.

11. A. Pelczynski, On B-spaces containing subspaces isomorphic to the space $c_{0}$, Bull. Acad. Polon. Sci. 5 (1957), 797-798.

12. - On the isomorphism of the spaces $M$ and $m$, Bull. Acad. Polon. Sci. 6 (1958), 695-696.

13. - Projections in certain Banach spaces, Studia Math. 19 (1960), 209-228.

14. A. Pelczynski and Z. Semadeni, Spaces of continuous functions (III), Studia Math. 18 (1959), 211-222.

15. A. Pelczynski, On strictly singular and strictly cosingular operators I: Strictly singular and strictly cosingular operators in $C(S)$, Bull. Acad. Polon. Sci. 11 (1963), 379-386.

UNIVERSITY OF TEXAS AND

Pennsylvania State University 